\title{
Mecanismos para la Protección de los Derechos de Hijos e Hijas con Madres Privadas de la Libertad
}

\author{
1 Catherine Pérez Martínez ${ }^{2}$ Karolayn Ramírez Arredondo ${ }^{3}$ Astrid Milena Calderón \\ Cárdenas
}

RESUMEN Esta investigación busca analizar los ordenamientos jurídicos de Colombia y México a través del derecho comparado, con el fin de identificar aquellos mecanismos existentes para la protección de los derechos de los hijos de madres privadas de la libertad, quienes desde temprana edad viven en los centros penitenciarios, en concordancia con las Reglas de Bangkok que protegen los derechos de las mujeres recluidas así como los de sus hijos; este documento se realiza a través de tres momentos, descriptivo, comparativo y analítico, enfocando la investigación en la normatividad y la jurisprudencia de los países objeto de estudio y en la doctrina para describir mejor la problemática en materia carcelaria que enfrentan las dos naciones, encontrando en conclusión que los mecanismos existentes en Colombia son el derecho de petición y la acción de tutela y en el caso de México el derecho de petición y la queja.

Centros penitenciarios

Palabras

Derechos humanos

clave

Derechos de la niñez

Mecanismos de protección

Reglas Bangkok.

\footnotetext{
1 Autora: Estudiante de X semestre del Programa de Derecho de la Fundación Universitaria del Área Andina Seccional Pereira. Integrante del Semillero de Derecho Penitenciario y Carcelario, adscrito al Grupo de Investigación GEIS. Correo Institucional: cperez64@estudiantes.areandina.edu.co

2Autora: Estudiante de X semestre del Programa de Derecho de la Fundación Universitaria del Área Andina Seccional Pereira. Integrante del Semillero de Derecho Penitenciario y Carcelario, adscrito al Grupo de Investigación GEIS. Correo Institucional: kramirez20@estudiantes.areandina.edu.co

3 Coautora: Docente - Abogada Especialista en Derecho Administrativo, Magister en Historia y Doctoranda en Educación; directora del Semillero de Derecho Penitenciario y Carcelario, adscrito al Grupo de Investigación GEIS de la Facultad de Derecho de la Fundación Universitaria del Área Andina Seccional Pereira. Correo: amcalderon@areandina.edu.co
} 


\title{
Mechanisms for the Protection of the Rights of Sons and Daughters with Mothers Deprived of Liberty
}

\begin{abstract}
This research seeks to analyze the legal systems of Colombia and Mexico through comparative law, in order to identify existing mechanisms for the protection of the rights of the children of mothers deprived of liberty, who from an early age live in the centers. penitentiaries, in accordance with the Bangkok Rules that protect the rights of detained women as well as those of their children; This document is carried out through three moments, descriptive, comparative and analytical, focusing the research on the regulations and jurisprudence of the countries under study and on the doctrine to better describe the problems in prison matters faced by the two nations, finding In conclusion, the existing mechanisms in Colombia are the right of petition and the protection action and in the case of Mexico, the right of petition and complaint
\end{abstract}

Prisons

KEYWORDS Human Rights Child Rights Protection Mechanisms Bangkok Rules. 


\section{INTRODUCCIÓN}

El cuidado de los niños y niñas es primordial en el mundo de hoy, los derechos de la niñez deben prevalecer en todas las naciones del mundo, en este documento se analiza la problemática mencionada a través del derecho comparado entre Colombia y México, países que comparten algunas semejanzas en sus ordenamientos jurídicos, así como en las problemáticas que enfrentan en materia carcelaria, tales como la vulneración masiva de DDHH y fundamentales.

Estos asuntos han sido previamente estudiados por diferentes entidades colombianas y mexicanas, tales como el ICBF, el INPEC y desde la academia en el caso colombiano, por su parte en México, es tema de investigación en la Comisión Nacional de los Derechos Humanos y por doctrinantes estudiosos de dicha situación, es por ello que se convierte en una investigación de interés para identificar desde la normatividad y la jurisprudencia, cuáles son los mecanismos para la protección de los derechos de los niños y niñas que residen con sus madres en centros de reclusión, donde es menester resaltar la importancia de que los infantes sean protegidos en todo momento.

El propósito se cumple por medio de un trabajo documental, en un momento descriptivo, comparativo y analítico, donde se indaga principalmente la función que cumplen los Estados para velar por su bienestar, investigación en la que se comparan los mecanismos colombianos y mexicanos para la protección de DDHH con las Reglas de Bangkok enfocadas principalmente en el tratamiento de las reclusas y por consiguiente de sus hijos e hijas.

\section{MATERIALES Y MÉTODOS}

Este proyecto se realiza a través de un minucioso estudio por medio de una investigación documental, con enfoque cualitativo, de acuerdo con lo anterior la presente investigación se elaboró a través de la metodología de derecho comparado para establecer semejanzas y diferencias, con la comparación de ordenamientos jurídicos de Colombia y México frente a las Reglas Bangkok, con uso de fuentes certificadas en la Web, Bases de datos de la Fundación Universitaria del Área Andina y recursos de libre acceso para investigadores, textos normativos de México y Colombia, Jurisprudencia ubicada en la Relatoría de las Altas Cortes y doctrina de estudiosos en la materia. La selección del tema acerca de los mecanismos de protección de los derechos de hijos e hijas de mujeres privadas de la libertad como parte se realizó de un macroproyecto del Semillero de Derecho Penitenciario y Carcelario de la Fundación Universitaria del Área Andina durante el verano de investigación del programa Delfín en conjunto con estudiantes provenientes de México razón por la cual se seleccionó este país dada su similitud en los ordenamientos jurídicos. 


\section{RESULTADOS}

En Colombia y México existe una crisis carcelaria reconocida por las Altas Cortes, la falta de protección total de las necesidades básicas, para las madres y sus hijos e hijas en los centros de reclusión, son muestra de dicha problemática que debe ser atendida de manera inmediata por parte de cada Estado. Ambos países coincidieron en que la edad de permanencia de los niños y niñas con sus madres en los establecimientos carcelarios es de cero a tres años y tanto en Colombia como en México los centros de reclusión requieren un cambio de fondo, en el que la infraestructura y la calidad de los servicios sean óptimos.

Los mecanismos de protección como el derecho de petición y la acción de tutela en Colombia o el derecho de petición y la queja en el caso de México se han establecido para garantizar derechos, pero lo que se encontró en realidad es la vulneración continúa, identificando que existen falencias en los ordenamientos y que se requiere la atención de los gobiernos de cada nación para que los mecanismos sean realmente efectivos y sean conocidos por la ciudadanía. En Colombia se ha tomó la medida excepcional del estado de cosas inconstitucional por parte de las Altas Cortes, en México no se habló de estas medidas para atender la vulneración masiva de derechos fundamentales, es evidente que los Estados no están cumpliendo con lo establecido en las Reglas de Bangkok, pues la violación de derechos de los niños y niñas, de las madres gestantes y lactantes continúa.

Colombia y México se muestran como países muy similares en su normatividad, en cuanto a la protección de los derechos de la infancia, pero se distancian cuando se analizó el tema desde lo jurisprudencial; por otra parte los Estados aún tienen mucho por hacer para detener la vulneración constante de los Derechos Humanos en los centros de reclusión, donde se están viendo afectados los intereses de los niños y niñas, quienes se encuentran viviendo en estos lugares en una etapa que define su desarrollo, físico, mental y psicológico, razón por la cual la aplicación de las reglas de Bangkok debe hacerse sin omitir ningún detalle, para que sus vidas sean más dignas y sus derechos sean respetados.

\section{DISCUSIÓN}

La existencia de mecanismos para proteger derechos fundamentales en ambos Estados existe, pero no está siendo efectiva ya que la crisis continúa y los infantes son los que están viendo afectadas sus garantías, en lugares con infraestructura deficiente, condiciones insalubres, trato poco digno y lejos de lo que sería una infancia plena y normal.

Se evidenció que a nivel normativo existen múltiples artículos que hablan sobre el tema, desde la jurisprudencia existe un reconocimiento de la problemática pero todo esto se está quedando solo 
en el papel sin medidas eficaces, por ello esta investigación tiene un impacto social, al ser de interés común para quienes desean conocer más o para los directamente afectados y un impacto jurídico al abordar temáticas como la normatividad y jurisprudencia en relación con las reglas Bangkok en un comparativo que complementa otras investigaciones en la materia.

Tanto en Colombia como en México se vive una crisis en materia penitenciaria. A nivel normativo, Colombia y México concuerdan con las Reglas de Bangkok, pero no se aplican a cabalidad, aunque se reconoce la prevalencia de los derechos de los infantes y la crisis carcelaria.

Los mecanismos en Colombia son el Derecho de Petición y la Acción de Tutela, en México son el Derecho de Petición y la Queja. Colombia posee una medida excepcional creada desde la Jurisprudencia para la protección de los derechos humanos en los centros penitenciarios, en México no se ha establecido medida alguna.

En los dos países comparados prevalecen los derechos de los niños y niñas, teniendo como base el Interés Superior de la Niñez y se debe atender la crisis carcelaria con prontitud pues las normas están solo en el papel.

\section{Comparación:}

Tabla 1

Comparación de las Reglas de Bangkok frente los mecanismos jurídicos para la protección de los niños y niñas en centros penitenciarios

\begin{tabular}{|c|c|c|c|}
\hline Comparación & $\begin{array}{l}\text { Reglas de } \\
\text { Bangkok }\end{array}$ & Colombia & México \\
\hline $\begin{array}{l}\text { Normatividad } \\
\text { general que protege } \\
\text { los derechos de los } \\
\text { hijos e hijas que } \\
\text { residen con sus } \\
\text { madres en los } \\
\text { centros } \\
\text { penitenciarios }\end{array}$ & $\begin{array}{l}\text { Reglas alusivas al } \\
\text { tema tratado: } 5 \text {, } \\
14,21,22,23,42,48 . \\
49,50,51,52 . \\
(\mathrm{CNDH}, 2018)\end{array}$ & $\begin{array}{l}\text { Const. Política: } \\
\text { Artículos } 44 \text { y 13: } \\
\text { Legislación: Ley } \\
\text { 1098 de 2006 Arts. 8, } \\
\text { 9, 10, 11,17,18, 20, } \\
\text { 21, 22, 23, 24, 26, 27, } \\
\text { 29, 30, 33,37. (Código } \\
\text { de Infancia y } \\
\text { Adolescencia, 2019) } \\
\text { Código Penitenciario } \\
\text { y Carcelario: Art. } \\
\text { 153 Mod. Por el Art. } \\
\text { 88 de la Ley 1709 de } \\
\text { 2014. (2018) }\end{array}$ & $\begin{array}{l}\text { Const. Política: Arts. } 1 \\
\text { y 4. (Constitución } \\
\text { Estados Unidos } \\
\text { Mexicanos, 1917) Ley } \\
\text { General de los } \\
\text { Derechos de Niñas, } \\
\text { Niños y Adolescentes: } \\
\text { Arts. 1, 2, 23. } \\
\text { Ley General de } \\
\text { Prestación de } \\
\text { Servicios para la } \\
\text { Atención Infantil } \\
\text { (Comisión Nacional de } \\
\text { Derechos Humanos } \\
\text { México, 2018) } \\
\text { Ley Nacional de } \\
\text { Ejecución Penal: Art. }\end{array}$ \\
\hline
\end{tabular}




\begin{tabular}{|c|c|c|c|}
\hline & & & $\begin{array}{l}\text { 36. (Congreso General } \\
\text { De Los Estados Unidos } \\
\text { mexicanos, 2016) }\end{array}$ \\
\hline \multicolumn{4}{|c|}{$\begin{array}{l}\text { Semejanzas con las Reglas Bangkok: } \\
\text { Colombia: A nivel Constitucional los derechos de los niños prevalecen como en las Reglas } \\
\text { Bangkok. } \\
\text { - La ley de infancia y adolescencia y el Código Penitenciario y Carcelario colombiano, protegen } \\
\text { a las madres gestantes y lactantes. México: Salvaguardar los derechos de la infancia, como una } \\
\text { obligación fundamental. La L.G.D.N.N.A concuerda con el reglamento. En cuanto a la Ley } \\
\text { General de Prestación de Servicios para la Atención Integral Infantil: calidad de vida, igualdad, } \\
\text { y protección plena a niños y niñas. Diferencias con las Reglas Bangkok: En ambos países no } \\
\text { existen leyes que desarrollen el contenido de las reglas de Bangkok. }\end{array}$} \\
\hline $\begin{array}{l}\text { Mecanismos para la } \\
\text { protección de los } \\
\text { derechos r res de } \\
\text { niños(as) en centros } \\
\text { carcelarios. }\end{array}$ & $\begin{array}{lr}\text { Las reglas } & \text { de } \\
\text { Bangkok sirven de } & \\
\text { guía, } & \text { los } \\
\text { mecanismos } & \text { de } \\
\text { protección } & \\
\text { dependen de } & \text { cada } \\
\text { país. } & \\
\end{array}$ & $\begin{array}{l}\text { Derecho de Petición: } \\
\text { (Const., 1991, Art.23). } \\
\text { Acción de Tutela: } \\
\text { Art. 86 Const. 1991. } \\
\text { (Constitución Política } \\
\text { de Colombia, 1991) }\end{array}$ & $\begin{array}{l}\text { Derecho de Petición } \\
\text { Artículo 8 Const. } \\
\text { Queja: de la Comisión } \\
\text { Nacional de los } \\
\text { Derechos Humanos. }\end{array}$ \\
\hline \multicolumn{4}{|c|}{$\begin{array}{l}\text { Semejanzas: En México y Colombia los mecanismos existentes para la protección de los } \\
\text { derechos de la niñez, buscan que se dé cumplimiento al reglamento, si se decide a favor del niño } \\
\text { o niña. } \\
\text { México: A través de los mecanismos creados para la protección de DDHH, en México se busca } \\
\text { salvaguardar las garantías de los niños y niñas que viven con sus madres en los centros de } \\
\text { reclusión cumpliendo las reglas. } \\
\text { Diferencias: Colombia: El reglamento internacional debe cumplirse en todo momento, sin } \\
\text { embargo, aún se dan casos en los que se desacatan los fallos o se niega el amparo del derecho. } \\
\text { México: Actualmente existen diversas situaciones que vulneran sus derechos, las cuales se dan } \\
\text { por la misma crisis carcelaria que existe en el país, vulnerando el reglamento. }\end{array}$} \\
\hline
\end{tabular}

\section{Tabla 2}

Comparación de las Reglas de Bangkok frente a las medidas para la protección de los niños y niñas en centros penitenciarios

\begin{tabular}{|l|l|c|c|}
\hline Comparación & $\begin{array}{l}\text { Reglas de } \\
\text { Bangkok }\end{array}$ & $\begin{array}{c}\text { Jurisprudencia } \\
\text { Colombia }\end{array}$ & $\begin{array}{c}\text { Jurisprudencia } \\
\text { México }\end{array}$ \\
\hline $\begin{array}{l}\text { Pronunciamiento } \\
\text { s de las Altas } \\
\text { Cortes. }\end{array}$ & $\begin{array}{l}\text { Reglas: 5, 14, 21, } \\
\text { 22, 23, 42, 48, 49, }\end{array}$ & $\begin{array}{c}\text { Corte } \\
\text { Constitucional: } \\
\text { Sentencia T-287 de }\end{array}$ & $\begin{array}{c}\text { Suprema Corte de } \\
\text { Justicia. }\end{array}$ \\
\hline
\end{tabular}




\begin{tabular}{|c|c|c|c|}
\hline & $\begin{array}{l}\text { 50, 51, } 52 \text { (CNDH, } \\
2018)\end{array}$ & $\begin{array}{c}\text { 2018. (Corte } \\
\text { Constitucional } \\
\text { Colombia, 2018) }\end{array}$ & $\begin{array}{l}\text { Amparo en Revisión } \\
644 / 2016 . \text { (Suprema } \\
\text { Corte de Justicia de la } \\
\text { Nación, México, 2016) }\end{array}$ \\
\hline \multicolumn{4}{|c|}{$\begin{array}{l}\text { Semejanzas: Colombia: Las Altas Cortes reconocen la prevalencia de los derechos de la } \\
\text { infancia tal como lo hacen las Reglas de Bangkok. Las Reglas de Bangkok destinadas para } \\
\text { la protección de la niñez, son reconocidas desde la jurisprudencia colombiana. México: El } \\
\text { contacto madre e hijo o hija es fundamental, lo reconocen las Altas Cortes mexicanas razón } \\
\text { por la cual buscan dar cumplimiento a reglas. La jurisprudencia resalta la prevalencia del } \\
\text { interés superior de la niñez tal como lo hace el reglamento y no se encuentran diferencias en } \\
\text { los dos países con relación al Reglamento. }\end{array}$} \\
\hline \multicolumn{4}{|c|}{$\begin{array}{l}\text { Diferencias: Colombia: El país se distancia de lo establecido en cada una de las reglas para } \\
\text { la protección de la niñez, dada la crisis carcelaria que ya fue reconocida por las Altas Cortes. } \\
\text { México Las Altas Cortes reconocen que los centros penitenciarios "padecen carencias } \\
\text { fundamentales en servicios e infraestructura. Semejanza: Tanto México como Colombia } \\
\text { incumplen de cierto modo las Reglas de Bangkok, ya que coinciden en la crisis que enfrentan } \\
\text { en materia penitenciaria y que no ha sido atendida de manera eficiente. }\end{array}$} \\
\hline $\begin{array}{l}\text { Estado de cosas } \\
\text { Inconstitucional } \\
\text { Sí o No }\end{array}$ & $\begin{array}{l}\text { Todas las reglas se } \\
\text { dirigen a la } \\
\text { protección } \\
\text { derechos que se ven } \\
\text { afectados por la } \\
\text { problemática } \\
\text { carcelaria, la cual se } \\
\text { presenta } \\
\text { actualmente en la } \\
\text { mayoría de los } \\
\text { Estados } \\
\text { Americanos. }\end{array}$ & $\begin{array}{l}\begin{array}{l}\text { Sí: Desde } 1998 \text { fue } \\
\text { declarado } \\
\text { en }\end{array} \\
\text { Sentencia T-153 de } \\
\mathbf{1 9 9 8} \text { (Corte } \\
\text { Constitucional } \\
\text { Colombia, 1998) , } \\
\text { ratificado en la T-388 } \\
\text { de } \mathbf{2 0 1 3} \text { y reiterado } \\
\text { por T-267 de 2015. } \\
\text { (Corte Constitucional } \\
\text { Colombia, 2015) }\end{array}$ & $\begin{array}{l}\text { No: no se encuentran } \\
\text { pronunciamientos que } \\
\text { hagan alusión al estado } \\
\text { de cosas } \\
\text { inconstitucional ni a } \\
\text { medidas excepcionales. }\end{array}$ \\
\hline
\end{tabular}

\section{SUGERENCIAS}

La presente investigación al ser netamente documental, se vería beneficiada con la elaboración de un trabajo de campo que permita explorar la problemática directamente en los centros penitenciarios donde los niños y niñas conviven con sus madres recluidas tanto en Colombia como en México, transformando la investigación hacía un enfoque mixto al obtener datos estadísticos de carácter cuantitativo que darían resultados más precisos, que faciliten promover la protección de derechos de niños y niñas, que viven su primera infancia en centros de reclusión.

Lo anterior requiere de un compromiso y apoyo de los Estados iniciando por sus gobiernos locales a través de la implementación de políticas públicas y la enseñanza a la ciudadanía acerca de los principales mecanismos para la protección de los DDHH. 


\section{AGRADECIMIENTOS}

Agradecemos a la docente y coautora de esta investigación, Astrid Milena Calderón Cárdenas, por creer en nosotras como estudiantes investigadoras y darnos la oportunidad de ser partícipes en un tema tan importante en el cual desde la Academia se pueden realizar importantes aportes para la sociedad en general y para los estudiantes de ciencias jurídicas, agradecimiento extendido a la Fundación Universitaria del Área Andina en su esfuerzo por fomentar el interés en asuntos investigativos que incentivan a crear más conocimiento en áreas jurídico- sociales, finalmente agradecer a nuestras familias Pérez Martínez y Ramírez Arredondo por el apoyo incondicional durante este proceso.

REFERENCIAS
1. CNDH México. (2018). Reglas de las Naciones Unidas para el Tratamiento de las Reclusas. Recuperado de https://bit.ly/2wUFN8Z

2. Código de la Infancia y la Adolescencia [Código]. (2019). Avance Jurídico Casa Editorial Ltda. Recuperado de https://www.icbf.gov.co/cargues/avance/docs/ley_1098_2006.htm

3. Código Penitenciario y Carcelario [Código]. (2019). Avance Jurídico Casa Editorial Ltda. Recuperado de: http://www.secretariasenado.gov.co/senado/basedoc/ley_0065_1993 _pr003.html\#152

4. Comisión Nacional de Derechos Humanos México. (2018). Ley General de Prestación de Servicios para la Atención, Cuidado y Desarrollo Integral Infantil. Recuperado de https://bit.ly/3d1WVu5

5. Congreso General De Los Estados Unidos Mexicanos. (16 de junio de 2016) Ley Nacional de Ejecución Penal. Recuperado de: https://bit.ly/33rxv4D

6. Constitución política de Colombia [Const.] (1991). Recuperado de https://bit.ly/2QivzpD

7. Constitución Política de los Estados Unidos Mexicanos [Const.] (1917) Recuperado de http://www.diputados.gob.mx/LeyesBiblio/pdf/1_060619.pdf

8. Corte Constitucional de Colombia. (16 de diciembre de 2015) Sentencia T-762 de 2015 [MS. GLORIA STELLA ORTIZ DELGADO]

9. Corte Constitucional de Colombia. (1998). Sentencia T-153 de 1998. [M.P. Eduardo Cifuentes Muñoz].

10. Corte Constitucional de Colombia. (2018). Sentencia T-287 de 2018. [M.P. Carlos Bernal Pulido].

11. Suprema Corte de Justicia de la Nación México. Amparo en Revisión 644/2016 [M.P. María Arturo Zaldívar Lelo de Larrea]. 